\title{
Characterizing Magnetic Anisotropy in Amorphous Metal Films Using Tilted Fluctuation Electron Microscopy
}

Ellis Kennedy ${ }^{1}$, Alejandro Ceballos ${ }^{2,3}$, Frances Hellman ${ }^{1,2,3}$, Colin Ophus ${ }^{4}$, Mary Scott ${ }^{1,4}$

1. Department of Materials Science and Engineering, University of California, Berkeley, Berkeley, CA, USA.

2. Department of Physics, University of California, Berkeley, Berkeley, CA, USA.

3. Materials Sciences Division, Lawrence Berkeley National Lab, Berkeley, CA, USA.

4. The Molecular Foundry, Lawrence Berkeley National Lab, Berkeley, CA, USA.

Compounds consisting of transition metals and rare earth elements often exhibit desirable magnetic properties for application in memory devices. For example, sputtered amorphous $\mathrm{Tb}_{\mathrm{x}} \mathrm{Co}_{1-\mathrm{x}}(a-\mathrm{Tb}-\mathrm{Co})$ thin films are magnetically anisotropic under certain growth conditions, with the easy axis perpendicular to the plane of the film [1]. This magnetic anisotropy depends on growth conditions, composition, and postgrowth heat treatment. Although it is thought that the origin of the magnetic anisotropy is the single ion anisotropy of $\mathrm{Tb}$ together with an anisotropic local structure induced by the growth process, how the magnetic properties of this material depend on structure, and why it depends on both growth temperature and evolves with annealing, are unknown $[2,3]$. Due to the lack of long-range order in $a$-Tb-Co and other disordered materials, it is difficult to relate the changes in magnetic properties to changes in structure. Although X-ray diffraction has not shown significant changes in short-range order in $a$-Tb-Co, EXAFS studies have measured structural changes in a similar system with strong out-of-plane anisotropy, $a$-Tb$\mathrm{Fe}[4,5]$. The enhanced Tb-Fe correlations, as well as changes in interatomic spacing in the in-plane vs. out-of-plane directions, observed in [4] and [5] indicate that structural features are indeed correlated to the magnetic anisotropy in these systems. To better understand the relationship between structure, sample growth and treatment, and magnetic properties in these systems, it is desirable to measure the local ordering and symmetry in the 2-4 $\mathrm{nm}$ range in structurally and magnetically anisotropic systems.

Fluctuation electron microscopy (FEM) is a focused-probe, scanning nanodiffraction technique that is sensitive to medium range order in disordered materials (Fig. 1). By measuring a series of diffraction patterns and calculating the variance in intensity as a function of spatial frequency, FEM has been used to determine the relative amount of medium range on the size scale of the probe used [6]. Typically, this method is used to detect changes between samples, relating differences in variance and average bond length to changes in MRO arising from processing or sample compositions. Additionally, the FEM variance data can be used as a constraint in reverse Monte Carlo calculations [7] to solve for atomic coordinates. This method has been successfully applied to understand structural evolution of a variety of amorphous systems. However, a drawback of FEM is that it typically assumes an isotropic sample. Therefore, we have extended FEM to probe directionally-dependent properties of anisotropic materials by tilting the sample during sequential data scans. Since diffracted intensity depends most strongly on inplane periodicities (i.e., periodic structures that lie in the plane perpendicular to the beam direction), this method probes in-plane and out-of-plane structures as the sample's tilt is changed. This allows for comparison to in-plane and out-of-plane variance as a way to determine differences in medium range order in these directions.

We have performed tilted FEM experiments on $a$-Tb-Co samples with low and high out-of-plane magnetic anisotropy. To calculate precise variance values, a fitting protocol was developed to remove ellipticity resulting from imperfect microscope alignments. Fitted and corrected diffraction patterns were analyzed 
to determine differences in variance as a function not just of spatial frequency, but also with respect to scattering angle. Ultimately, angle-dependent variance values can be used to determine the structural changes that are correlated to changes in magnetic anisotropy in this system. By relating changes in MRO to changes in short range order, the atomic-scale structural origins of magnetic anisotropy in these amorphous metal films can be understood [8].

References:

[1] T. Ueno et al, Journal of Physics D: Applied Physics, 49 (2016), p. 205001.

[2] S. Yoshino et al, Japanese Journal of Applied Physics, 23 (1984), p. 188.

[3] F. Hellman and E. M. Gyorgy, Physical review letters, 68.9 (1992), p. 1391.

[4] V.G. Harris et al, Physical review letters, 69 (1992), p. 1939.

[5] V.G. Harris et al, Physical Review B, 49 (1994), p. 3637.

[6] P.M. Voyles and D.A. Muller, Ultramicroscopy, 93 (2002), p. 147.

[7] J.J. Maldonis, J. Hwang, and P.M. Voyles, Computer Physics Communications, 213 (2017), p. 217.

[8] This project is supported by the U.S. Department of Energy, Office of Science, Office of Basic Energy Sciences, Materials Sciences and Engineering Division under Contract No. DE-AC02-05CH11231 within the Non-Equilibrium Magnetic Materials Program. Work at the Molecular Foundry was supported by the Office of Science, Office of Basic Energy Sciences, of the U.S. Department of Energy under Contract No. DE-AC02-05CH11231.

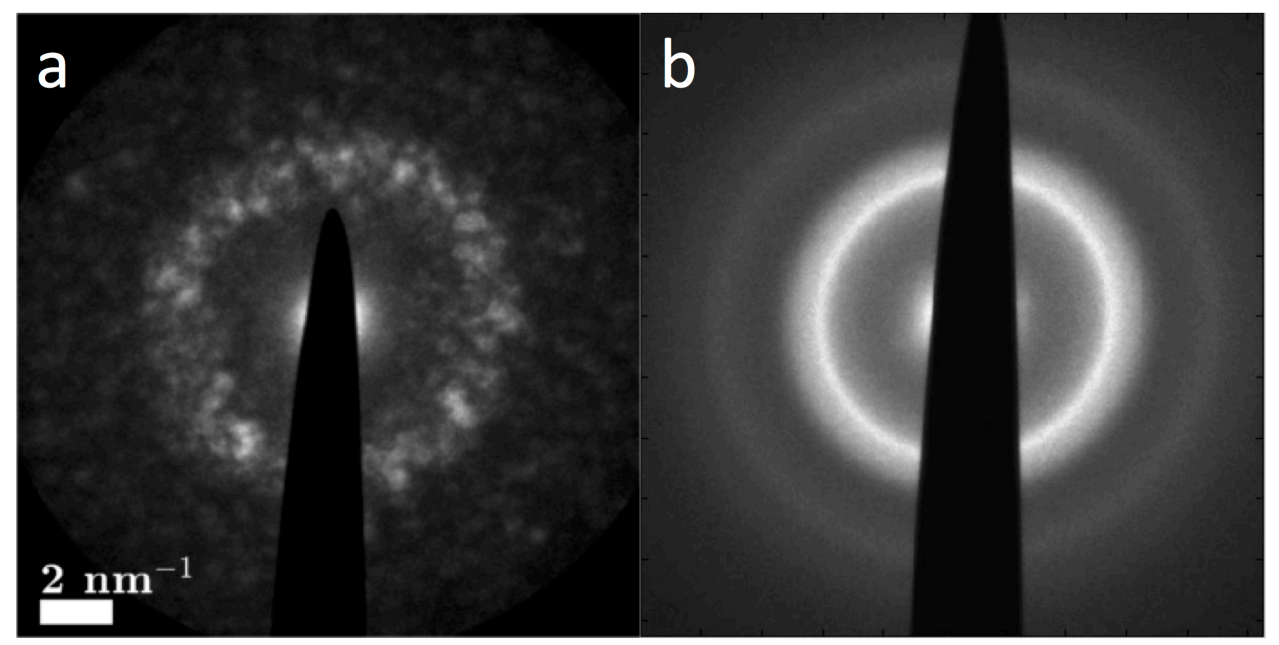

Figure 1. Comparison of (a) nanodiffraction and (b) parallel beam diffraction. In (a), confining the probe to a small region $(2 \mathrm{~nm})$ gives sensitivity to small regions of ordering. In (b) such features are averaged over. 